\title{
Effectiveness of a dietary intervention on macronutrient intake, lean mass and strength gains in males participating in a supervised resistance training program
}

\author{
Jonathan Oliver ${ }^{*}$, Michelle Mardock, Andrew Jagim, Adam Sanchez, Julie Kresta, Stephen Crouse, Richard Kreider
}

From International Society of Sports Nutrition: 8th Annual ISSN Conference and Expo

Las Vegas, NV, USA. 24-25 June 2011

\section{Background}

ISSN recommendations for individuals involved in a general fitness program are to ingest $25-35 \mathrm{kcal} / \mathrm{kg} /$ day consisting of $3-5 \mathrm{~g} / \mathrm{kg}$ of carbohydrate and $\leq 30 \%$ of total calories from fat. Additionally, the ISSN recommends that individuals engaged in resistance-training should ingest $1.4-2.0 \mathrm{~g} / \mathrm{kg} / \mathrm{d}$ of protein and to ingest some protein after exercise. This study examined whether nutritional counseling and post-workout supplementation affects dietary intake during training.

\section{Methods}

Eleven trained men $(25 \pm 5$ yrs, $180 \pm 6 \mathrm{~cm}, 82 \pm 12 \mathrm{~kg}, 14 \pm 3$ $\%$ fat, training $7 \pm 4$ years, $3 \pm 2$ days/wk) were provided nutritional counseling by a dietitian prior to participating in a supervised resistance-training program (4 days/wk). A supplement containing $40 \mathrm{~g}$ carbohydrate, $20 \mathrm{~g}$ protein, and $3.5 \mathrm{~g}$ fat was provided post-exercise. Diet records were obtained at $0,3,7, \& 11$ weeks while DEXA determined body composition, 1RM bench press, and 1RM squat measurements were obtained at $0,4,8, \& 12$ wks. Data were analyzed by ANOVA with repeated measures and are presented as means \pm standard deviations.

\section{Results}

Nutritional counseling did not change energy intake (30.9 $\pm 5.5,36.4 \pm 9.6,35.0 \pm 10.2,33.1 \pm 6.1 \mathrm{kcal} / \mathrm{kg} /$ day; $\mathrm{p}=0.20$ ) or fat intake (34 $\pm 10,34 \pm 6,34 \pm 6,34 \pm 7 \% ; \mathrm{p}=0.97)$. Protein intake significantly increased from baseline $(1.7 \pm 0.4,2.4$ $\pm 0.8,2.3 \pm 0.6,2.4 \pm 0.5 \mathrm{~g} / \mathrm{kg} ; \mathrm{p}=0.002$ ) while carbohydrate

Exercise \& Sport Nutrition Lab, Texas A\&M University, College Station, TX 77843, USA intake significantly decreased (3.5 $\pm 1.2,3.3 \pm 0.6,2.8 \pm 1.2,2.3$ $\pm 0.9 \mathrm{~g} / \mathrm{kg} ; \mathrm{p}=0.02$ ); corresponding to an increase in percentage of protein $(22 \pm 6,26 \pm 3,28 \pm 10,29 \pm 6 \%$; $p=0.03)$ and a decrease in percentage of carbohydrates $(45 \pm 15,38$ $\pm 8,31 \pm 10,28 \pm 9 \% ; \mathrm{p}=0.003)$. After 4,8 and 12 weeks, respectively, a significant increase in lean mass was observed $(1.3 \pm 1.7,2.1 \pm 1.8,2.2 \pm 2.1 \mathrm{~kg}$; $\mathrm{p}=0.001)$ with no significant effect on body fat percentage $(14.3 \pm 2.7,15.0$ $\pm 3.3,14.7 \pm 3.5,15.1 \pm 3.5 \%$; $\mathrm{p}=0.34)$. Bench press $1 \mathrm{RM}(-2$ $\pm 6,3 \pm 6,9 \pm 5 \% ; \mathrm{p}=0.001)$ and squat $1 \mathrm{RM}(14 \pm 10,33 \pm 14$, $43 \pm 18 \%$; $=0.001$ ) increased from baseline.

\section{Conclusion}

Nutritional counseling prior to engaging in a resistancetraining program that included post exercise supplementation increased dietary protein intake and resulted in positive training adaptations despite a reduction in carbohydrate intake. Additional nutritional guidance may be necessary to ensure adequate carbohydrate intake particularly in athletes engaged in heavy training.

\section{Funding}

Supported by National Strength and Conditioning Association. Supplements provided by Cytosport ${ }^{\mathrm{TM}}$, Inc.

Published: 7 November 2011

doi:10.1186/1550-2783-8-S1-P20

Cite this article as: Oliver et al:: Effectiveness of a dietary intervention on macronutrient intake, lean mass and strength gains in males participating in a supervised resistance training program. Journal of the International Society of Sports Nutrition 2011 8(Suppl 1):P20. 